\title{
No Perisaccadic Mislocalization with Abruptly Cancelled Saccades
}

\author{
Jeroen Atsma, ${ }^{1}$ Femke Maij, ${ }^{1,2}$ Brian D. Corneil, ${ }^{3,4,5}$ and W. Pieter Medendorp ${ }^{1}$ \\ ${ }^{1}$ Donders Institute for Brain, Cognition and Behaviour, Radboud University Nijmegen, Nijmegen $6500 \mathrm{HB}$, The Netherlands, ${ }^{2}$ University of Birmingham, \\ School of Psychology, Birmingham B15 2TT, West Midlands, United Kingdom, and Departments of ${ }^{3}$ Physiology and Pharmacology, ${ }^{4}$ Psychology, and \\ ${ }^{5}$ Robarts Research Institute, Western University, London, Ontario N6A 5B7, Canada
}

Every saccadic eye movement that we make changes the image of the world on our retina. Yet, despite these retinal shifts, we still perceive our visual world to be stable. Efference copy from the oculomotor system to the visual system has been suggested to contribute to this stable percept, enabling the brain to anticipate the retinal image shifts by remapping the neural image. A psychophysical phenomenon that has been linked to this predictive remapping is the mislocalization of a stimulus flashed around the time of a saccade. If this mislocalization is initiated by saccade preparation, one should also observe localization errors when a saccade is planned, but abruptly aborted just before its execution. We tested this hypothesis in human subjects using a novel paradigm that combines a flash localization task with a countermanding component that occasionally requires saccade cancellation. Surprisingly, we found no trace of mislocalization, even for saccades cancelled close to the point of no return. This strongly suggests that the actual execution of the saccade is a prerequisite for the typical localization errors, which rejects various models and constrains neural substrates. We conclude that perisaccadic mislocalization is not a direct consequence of saccade preparation, but arises after saccade execution when the flash location is constructed from memory.

Key words: countermanding; perisaccadic mislocalization; remapping; saccade preparation; visual stability

\section{Introduction}

Saccadic eye movements quickly reposition our line of sight to scan the world around us. During saccades, the image of the world shifts on our retina. Nevertheless, we perceive our visual world as being stable, which suggests that representation of the visual world is integrated over multiple saccades. In 1867, von Helmholtz proposed that the brain achieves visual stability by using a copy of a movement command-the efference copy-to adjust perception for the corresponding eye movement (von Helmholtz, 1925). In essence, using the efference copy, the brain differentiates sensory information arising from its own actions from those that arise from the environment.

Today there is accumulating neurophysiological evidence that the brain incorporates the efference copy of a saccade to achieve visual stability (for review, see Wurtz et al., 2011). For example, Morris et al. (2012) showed previously that updating of the cor-

\footnotetext{
Received Nov. 12, 2013; revised Feb. 7, 2014; accepted Feb. 24, 2014.

Author contributions: J.A., F.M., B.D.C., and W.P.M. designed research; J.A. performed research; J.A. analyzed data; J.A., F.M., B.D.C., and W.P.M. wrote the paper.

This work was supported by an internal grant from the Donders Centre for Cognition; European Research Council Grant EU-ERC 283567, EU-FP7-FET SpaceCog Grant 600785, and Netherlands Organisation for Scientific Research (NW0) Vici Grant 453-11-001 to W.P.M.; operating grants from the Natural Sciences and Engineering Research Council of Canada and Canadian Institutes of Health Research Grant MOP 93796 to B.D.C.; and NWO Rubicon Grant 446-10-015 and NWO Veni Grant 451-12-009 to F.M. We thank Dr. Koppen for fruitful discussion about the statistical approaches taken in this study.

The authors declare no competing financial interests.

Correspondence should be addressed to Jeroen Atsma, Radboud University Nijmegen, Donders Centre for Cognition, P.0. Box 9104, 6500 HE Nijmegen, the Netherlands. E-mail: j.atsma@donders.ru.nl.

DOI:10.1523/JNEUROSCI.4773-13.2014

Copyright $\odot 2014$ the authors $\quad 0270-6474 / 14 / 345497-08 \$ 15.00 / 0$
}

tical representation of the eye position starts before saccade initiation. Furthermore, neurons in various cortical and subcortical areas have retinotopic receptive fields that are not fixed to gaze, but shift in the direction of the saccade just before the eyes start to move. Such neurons have been identified in the lateral intraparietal area (LIP; Duhamel et al., 1992), the frontal eye field (FEF; Umeno and Goldberg, 1997), the superior colliculus (SC; Walker et al., 1995), and earlier visual areas like V4 (Moore et al., 1998), V3, and V2 (Nakamura and Colby, 2002).

A psychophysical phenomenon that has been linked to shifting receptive fields is the systematic mislocalization of brief stimuli presented around the time of a saccade (Ross et al., 1997, Burr and Morrone, 2010; Hamker et al., 2008). Such perisaccadic localization errors can begin $\sim 100$ ms before saccade onset, and peak when flashes are presented around saccade onset. It has been suggested that the mislocalization is driven, at least in part, by presaccadic activity of neurons in the SC or FEF (Hamker et al., 2008). This implies that the mere preparation of a saccade may contribute to perisaccadic mislocalization (Hamker, 2005, 2008, 2011; Cicchini et al., 2013). If so, can perisaccadic mislocalization be observed when a saccade is planned, but ultimately aborted just before its execution?

To answer this question, we designed a novel paradigm that combines a mislocalization task with a countermanding component that occasionally requires saccade cancellation. The countermanding component has a refined theoretical architecture (Logan and Cowan, 1984), and an extensive literature describes the neurophysiology that underlies saccadic behav- 
ior (for review, see Schall and Godlove, 2012). This literature demonstrates that oculomotor preparation can be highly advanced on cancelled saccades; many saccade-related neurons in the SC and FEF exhibit increasing levels of activity before being abruptly curtailed (Hanes et al., 1998; Paré and Hanes, 2003; Brown et al. 2008), and oculomotor preparation when the head is unrestrained can even initiate orienting head movements on cancelled trials where the line of sight remains stable (Corneil and Elsley, 2005). Inclusion of the countermanding component therefore provides a test of whether preparation alone can drive mislocalization, independent of other perisaccadic or postsaccadic processes.

\section{Materials and Methods}

Eight naive human participants (five male, three female, aged 19-30 years) gave informed consent to take part in the experiment. All subjects had normal or corrected-to-normal visual acuity and were free of any known sensory, perceptual, or motor disorders. The study was part of a research program approved by the ethics committee of the Social Sciences Faculty of the Radboud University Nijmegen. Each subject participated in four experimental sessions of approximately $1 \mathrm{~h}$ each.

Experimental setup. Each subject sat in a dimly lit room (luminance, $\sim 0.06 \mathrm{~cd} / \mathrm{m}^{2}$ ) with their head supported by a chin rest. They operated a two-button computer mouse. Stimuli were controlled using a custom-written program in Delphi (Embarcadero) software. Visual stimuli were projected onto a screen using a projector (Sharp PG-M20X) running at 60 $\mathrm{Hz}$ with a resolution of $1024 \times 768$ pixels. The projection screen was placed $\sim 90 \mathrm{~cm}$ in front of the subject, creating a display with a visual field of $67 \times 52^{\circ}$. The top 10 rows of pixels were projected on a wall behind the projection screen, invisible to the subject, but detected by a photo diode to determine the precise onset of stimuli. Binocular eye position was recorded at $500 \mathrm{~Hz}$ using a head-mounted eye tracker (EyeLink II; SR Research). Because the paradigm is contingent on saccades, saccade onsets were detected and processed on-line using an eye velocity criterion of $150 \%$ s. A saccade was considered inhibited (i.e., cancelled) when the velocity did not reach $50 \%$ within $500 \mathrm{~ms}$ after target onset. A higher velocity threshold was used for detecting a saccade compared to detecting inhibition because otherwise sporadic small saccades could potentially confound the timing of to-be-localized flash. All stimuli were projected on a black background $\left(0.18 \mathrm{~cd} / \mathrm{m}^{2}\right)$.

Design issues. We combined a countermanding task with a flashlocalization task. The design of the paradigm was based on the following considerations. (1) The mislocalization effect should be substantial and should arise as early as possible relative to saccade onset. (2) Saccade reaction times (SRTs) should be highly predictable. (3) Saccade preparation should be encouraged as much as possible. We discuss the rationale behind each of these considerations in turn.

First, substantial mislocalization should start early relative to saccade onset to increase the chance of observing mislocalization even on cancelled saccades. To do this, we opted to use large saccade amplitudes $\left(20^{\circ}\right.$; Richard et al., 2009) and a low-contrast flash (Michels and Lappe, 2004) that was positioned near the fixation point (Richard et al. 2009; Maij et al., 2011a) and referenced to a continuously visible static ruler that pro- vided a strong visual reference (Lappe et al., 2000; Awater and Lappe, 2006). As intended, these factors produced a large mislocalization effect that started up to $100 \mathrm{~ms}$ before saccade onset (see Results).

Second, since by definition no saccade occurs on successfully cancelled stop trials, SRTs had to be as predictable as possible. Such predictability allows us to express the localization error relative to the SRT that would have been produced if the saccade had not been cancelled. To this end, we used a rhythmic $(2 \mathrm{~Hz})$ sequence of saccades in the beginning of each trial, which is known to reduce SRT variability (Maij et al., 2011a).

Third, to encourage saccade preparation as much as possible once the $2 \mathrm{~Hz}$ rhythm was established, the overall length of the saccade sequence was made unpredictable, so that subjects made in total four to seven saccades per trial. The last saccade of the sequence was the test trial, in which an imperative stop signal was either presented (two-thirds of all trials) or not. However, the probability of a stop signal occurring on a given saccade varied across the saccade sequence (one-sixth, two-ninths, one-third, and two-thirds for the fourth through seventh saccades, respectively). This feature ensured that saccades were usually required, with stopping required on a minority of most saccades within the sequence (i.e., the probability of stopping exceeded 0.5 only when the sequence was seven saccades long).

Paradigm. Figure $1 \mathrm{~A}$ depicts the course of a trial. A static white ruler $\left(60 \times 4.5^{\circ}, 165 \mathrm{~cd} / \mathrm{m}^{2}\right)$ was continuously present at the bottom of the screen. Subjects made the sequential saccades to targets (gray dot, size $0.8^{\circ}$, luminance $28.3 \mathrm{~cd} / \mathrm{m}^{2}$ ) regularly presented at $500 \mathrm{~ms}$ intervals. Saccade targets were presented at $20^{\circ}$ eccentricity. With the presentation of a new target, the previous target was rendered dark gray for $500 \mathrm{~ms}$, 
after which it completely disappeared. The final (probed) saccade in the sequence was always directed horizontally (leftward or rightward), whereas the other saccades were directed either horizontally or deviated $45^{\circ}$ from horizontal. In two-thirds of the trials, the final saccade was accompanied with an imperative stop signal. The stop signal was given by changing the color of the second last target (the current fixation point) to red. The second last target, or stop signal, and the last saccade target disappeared simultaneously $500 \mathrm{~ms}$ after the onset of the last saccade target. Near the time of the onset of the final saccade, either inhibited or not, a dark-green vertical bar $\left(0.4 \times 7.6^{\circ}, 0.32 \mathrm{~cd} / \mathrm{m}^{2}\right)$ was flashed for $16.7 \mathrm{~ms}$ (one frame). The flash was aligned vertically with the fixation and saccade target and positioned either in between the two targets (inbound flash; $+10^{\circ}$ ) or $10^{\circ}$ from fixation into the opposite direction (outbound flash; $-10^{\circ}$ ). The onset time of the flash was chosen randomly from the range of -150 to $+50 \mathrm{~ms}$ relative to the average SRT of the previous 10 trials. After disappearance of the last saccade target, the dark-green vertical bar reappeared on the far left of the display. Using a mouse, subjects moved it to a location where they had perceived the flash, which they confirmed by clicking the left mouse button. Subjects had to press the right mouse button when the flash was not perceived. The next trial then started.

We varied the stop signal delay (SSD), i.e., the time between the stop cue and the saccade target, using a one-up, one-down staircase procedure with a step size of $33.3 \mathrm{~ms}$ (two frames). As a result, the SSD fluctuated around the interval where subjects cancelled about half of the stop trials. Since two of three trials contained a stop signal, the numbers of go trials (those without a stop signal), noncancelled trials (i.e., where a saccade is made despite the stop signal), and cancelled trials were approximately equal.

Before the actual experiment started, subjects performed $\sim 120$ trials to familiarize themselves with the paradigm. The eye tracker was calibrated (using a nine-point grid) every time the program failed to detect a fixation, which signaled that the eye tracking error was $>3^{\circ}$ (on average, this happened every 120 trials). Every 50 trials, subjects were allowed to take a small break. Each subject completed one session of 300 and three sessions of 400 trials on separate days, resulting in 1500 trials per subjects. The total experiment lasted approximately $4 \mathrm{~h}$ per subject.

Data analysis. We performed off-line data analyses in Matlab (MathWorks). A saccade was defined as a period where velocity exceeds $50 \%$ with the SRT being the time between target and saccade onset. Trials were excluded based on the parameters of the last saccade of a sequence. Note that we conservatively rejected a high number of trials to reduce the chance that the observed patterns of perisaccadic mislocalization were confounded by the preparation of incorrect eye movements, a lack of subject vigilance, or blinks. Saccades with a very short $(<100 \mathrm{~ms})$ SRT were excluded because these were likely generated by anticipation, rather than being directed to the final saccade target $(7.6 \pm 2.1 \%$ and $4.7 \pm 1.5 \%$, mean $\pm \mathrm{SE}$, for the go trials and noncancelled trials, respectively). We also excluded very late ( $>400 \mathrm{~ms})$ SRTs on the basis of outliers $(5.9 \pm 1.4 \%$ and $3.5 \pm 0.9 \%)$. We also excluded saccades with amplitudes $<11^{\circ}$, since these saccades could be directed toward the flash $\left(\right.$ at $\left.10^{\circ}\right)$ rather than the saccade target $(13.6 \pm 3.3 \%$ and $30.8 \pm 3.1 \%)$. Furthermore, saccades were discarded when they deviated $>10^{\circ}$ from the correct direction $(6.8 \pm 1.2 \%$ and $11.5 \pm 2.2 \%)$, when the saccade was preceded by a blink that could interfere with perceiving the stop signal or flash $(0.8 \pm 0.6 \%$ and $0.4 \pm 0.2 \%)$, and when a saccade was absent $(7.9 \pm$ $1.9 \%$ of the go trials). In $\sim 8.0 \pm 1.7 \%$ of the go trials, $8.4 \pm 1.9 \%$ of the noncancelled trials, and $0.2 \pm 0.1 \%$ of the cancelled trials, the participant reported not having seen the flash, and these trials were also excluded from analysis. This means that, on average, $33.2 \pm 2.4 \%$ of the go trials, $46.7 \pm 2.7 \%$ of the noncancelled trials, and $0.2 \pm 0.1 \%$ of the cancelled trials, respectively, were discarded. We confirmed that this high percentage of rejected trials did not unduly influence the conclusion presented below, as similar results were obtained if we relaxed our exclusion criteria so that only $\sim 10 \%$ of go trials and noncancelled trials were discarded.

For the remaining trials, the localization response was defined in the horizontal direction as the difference between the indicated location and the fixation point. The response was signed positive toward the saccade target and signed negative to the opposite direction. The localization responses were further analyzed as a function of the flash onset time relative to the actual or predicted saccade onset. Mislocalization curves were created based on a running average convolving the errors with a Gaussian of $15 \mathrm{~ms}$ window width. The variance of the localization errors was computed using a sliding window of $40 \mathrm{~ms}$.

By definition, successfully cancelled stop trials lack a saccade onset time relative to which any mislocalization effect can be examined. To resolve this problem, we estimated the onset of the putative saccade in each cancelled trial, based on a model description of the SRT in the go trials, as if it was not aborted before execution. This linear regression model incorporated the mean SRT of the previous two trials; the SRTs of the second-last, third-last, and fourth-last saccades within the current sequence; whether the previous trial was a stop trial; whether the previous trial was a cancelled trial; whether the current saccade is leftward or rightward; and the current sequence length. We used the same model to predict the SRTs of both the noncancelled and successfully cancelled stop trials.

To characterize countermanding behavior, we computed inhibition functions that describe the probability of a noncancelled (i.e., executed) saccade on a stop trial as a function of SSD. We further computed an estimate of the time needed for saccade cancellation, i.e., the stop signal reaction time (SSRT), using the integration method (Logan, 1994). This method follows from the idea that a saccade escapes inhibition only when the associated SRT is smaller than SSD + SSRT. The probability that a saccade escapes inhibition for a given SSD (from the inhibition function) thus equals the probability that SRT $<$ SSD + SSRT. The go trials can serve as a baseline distribution of SRTs. When, for example, $20 \%$ of the saccades are not cancelled for a given SSD, these saccades can be represented by the $20 \%$ fastest saccades of the baseline distribution. The upper bound of these $20 \%$ fastest saccades marks the point where SRT $\approx$ SSD + SSRT. The SSRT can be obtained by simply subtracting the SSD from this SRT. Here, the SSRT was estimated at each SSD. To obtain a single SSRT value per subject, we averaged the SSRT across SSDs.

Statistical analyses. Both saccadic reaction time (in milliseconds) and saccade amplitude (in degrees) were compared between go trials and noncancelled trials, using two-tailed paired $t$ tests. Differences in localization errors (in degrees) and their variance (in degree squared) were examined using repeated-measures ANOVA, with flash location (inbound, outbound), trial type (go, noncancelled, cancelled), and time $(-150 \mathrm{~ms}, 0 \mathrm{~ms}$ relative to saccade onset) as independent factors. Differences were considered significant at $p<0.05$. Post hoc testing was performed as needed using $t$ tests. Finally, to address the potential for type II errors ("false negatives"), we also calculated the $95 \%$ confidence intervals (CIs) of the mislocalization effect in the cancelled trials, as appropriate.

\section{Results}

\section{Saccade behavior resembles previous} countermanding studies

Before examining how subjects localized the flash, we first examined whether their overall saccadic behavior varied across saccade sequence and whether such behavior conformed to the expectations from previous countermanding studies. Saccadic behavior is shown for a representative subject in Figure $1 B-D$. Figure $1 B$ shows that compared to the go trials (i.e., without stop signals), noncancelled stop trials have a shorter SRT. Across subjects, the SRT was significantly shorter on noncancelled (mean SRT \pm SE, $173 \pm 8 \mathrm{~ms})$ versus go trials $\left(185 \pm 8 \mathrm{~ms} ; t_{(7)}=4.8, p<0.005\right)$. Saccade amplitude was also slightly smaller for noncancelled saccades $\left(19.3 \pm 0.5^{\circ}\right)$ compared to saccades on go trials $(20.3 \pm$ $\left.0.5^{\circ} ; t_{(7)}=4.2, p<0.005\right)$. Both findings are consistent with previous countermanding results, since preparation on noncancelled saccades has to proceed on average slightly faster to escape inhibition (Logan, 1994), and because larger noncancelled saccades can be truncated midflight (Corneil and Elsley 2005). Figure $1 B$ also shows that SRT increased for longer sequence lengths, presumably due to the increasing probability of the appearance of a stop signal (see Materials and Methods). 

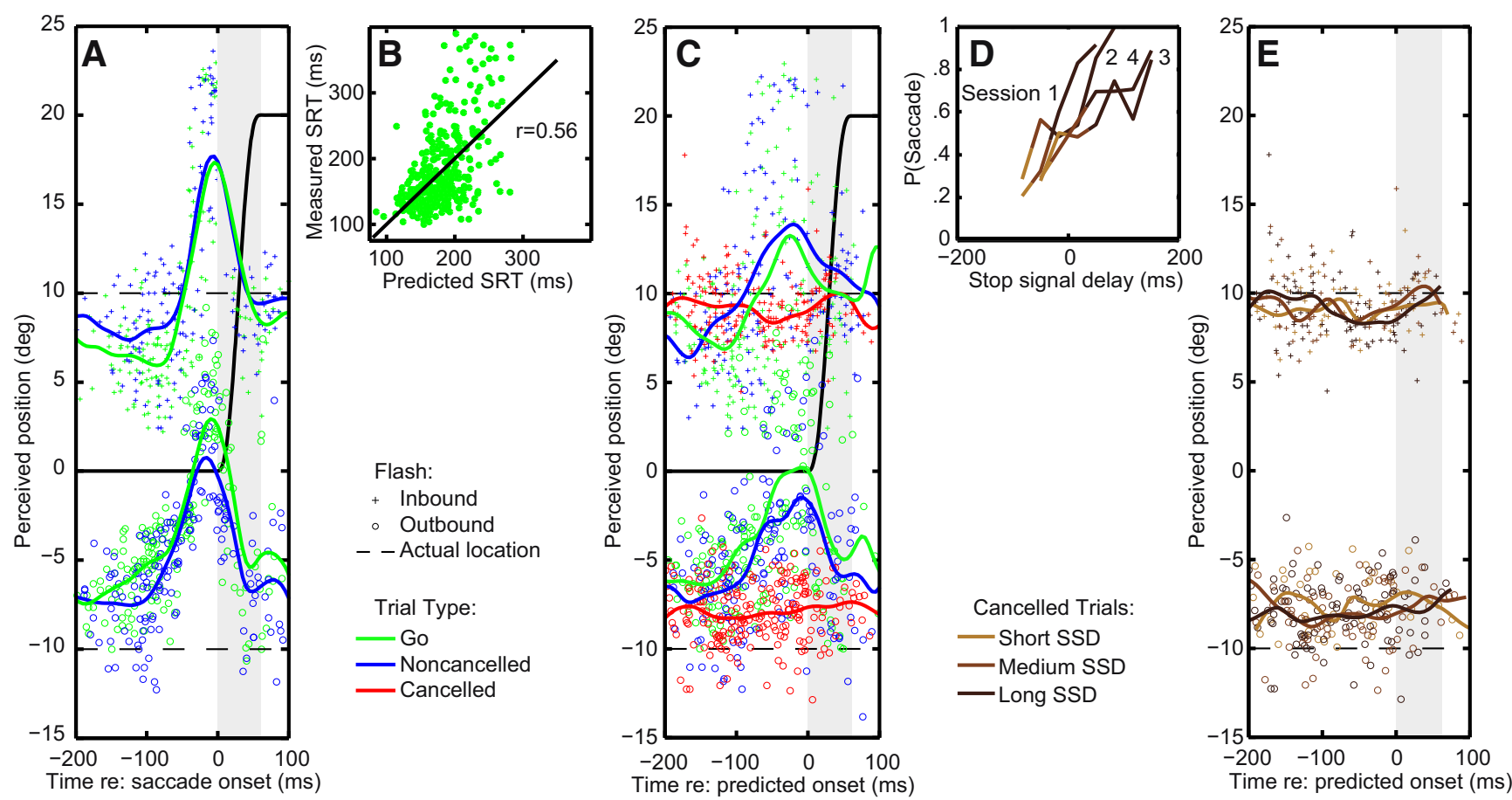

Figure 2. Single-subject analysis. $A$, Localization errors and mean curve (based on a Gaussian moving window of $15 \mathrm{~ms}$ ) as a function of flash onset relative to saccade onset. Go trials are in green; noncancelled trials are in blue. The black trace shows the planned saccade. The gray bar shows the mean saccade duration. Dashed lines indicate flash locations $\left(-10\right.$ and $\left.10^{\circ}\right) . B, M e a s u r e d ~ S R T$ versus predicted SRT, based on a linear regression model (see Materials and Methods), for the go trials. C, Localization errors and mean curve aligned to estimated SRT. Cancelled trials are in red. $\boldsymbol{D}$, Inhibition functions for each session: the probability of erroneously making a saccade as a function of the SSD. Cancelled trials were subdivided into three approximately equal-sized bins based on the SSD (short SSD, light red; medium SSD, red; long SSD, dark red). $\boldsymbol{E}$, Localization errors in the cancelled trials aligned to predicted saccade onset for the three subdivisions in SSD.

Inhibition functions for the representative subject are shown in Figure 1C. As expected, the probability of noncancelled saccades increased with SSD, because longer SSDs provide less time for saccade cancellation. The inhibition functions in Figure $1 C$, ordered by saccade sequence, shift rightward with longer sequence lengths, which mirrors the increasing SRTs (i.e., with a long SRT, inhibiting the saccade after a late stop signal is still possible). The mean SSRT across subjects was $165 \pm 37 \mathrm{~ms}$. For the representative subject in Figure $1 D$, the SSRT did not change systematically with sequence length. Also across subjects, a repeated-measures ANOVA confirmed that the SSRT is not influenced by sequence length $\left(F_{(3,5)}=2.59, p>0.05\right)$. In contrast, the SSRT showed a significant negative (linear) relationship with SSD, meaning that SSRTs were smaller for longer SSDs $\left(R^{2}\right.$ ranged from 0.966 to $0.992, p<0.005$ for all subjects). This observation is consistent with previous countermanding findings: only cancellation processes that proceed quickly can cancel saccades at longer SSDs (Logan and Cowan, 1984). Together, the countermanding analyses provide clear evidence that subjects are preparing the saccade, even if that saccade can be suddenly cancelled. The question that we now turn to is, how did subjects localize flashes on go trials without a stop signal, and on either cancelled on noncancelled trials with a stop signal?

\section{Flash localization when saccades are made}

Following previous studies, localization responses were analyzed as a function of the onset time of the flash relative to saccade onset. Figure $2 A$ illustrates the localization responses of the representative subject pooling across leftward and rightward saccades of $20^{\circ}$ amplitude, as plotted by the black curve. Flashes were presented at either the inbound $\left(+10^{\circ}\right)$ or outbound $\left(-10^{\circ}\right)$ location (Fig. 2A, dashed lines). The green and blue curves visualize the average trend of the go and noncancelled trials, respectively. Consistent with previous reports (Ross et al., 1997), clear localization errors are observed for targets flashed in the period of $\sim 100 \mathrm{~ms}$ before to $50 \mathrm{~ms}$ after the onset of the saccade. A repeated-measures ANOVA, with flash location (inbound, outbound), trial type (go, noncancelled), and time ( $-150 \mathrm{~ms} ; 0 \mathrm{~ms}$ relative to saccade onset) as independent variables revealed that across-subjects localization effects for the go and noncancelled trials did not differ significantly over time $\left(F_{(1,7)}=0.015\right.$, n.s.; Fig. $3 A$ ). This suggests that similar robust mislocalization is observed when a saccade is executed, regardless of the presence of a stop signal.

\section{Flash localization when saccades are cancelled}

In successful stop trials, the saccade is initially planned, but aborted before its execution. Since these trials lack a saccade onset time relative to which any mislocalization can be examined, we estimated when the saccade would have happened (predicted SRT) using a linear regression model (see Materials and Methods). The model revealed a significant correlation $(r=0.43 \pm$ 0.04 SE, $p<0.05$ for all subjects) between the predicted SRTs and the actual SRTs for go trials. Figure $2 B$ plots the measured SRT versus the predicted SRT of the go trials of the representative subject, showing a significant correlation coefficient $(r=0.56$, $p<0.05$ ). Note the model cannot account for the deviations from linearity at long SRTs (>300 ms). However, in these trials there is no systematic mislocalization since the flash is presented long before the saccade is made.

Based on the same model, we can also predict the SRTs of the noncancelled stop trials and the successfully cancelled stop trials. This allows a direct comparison of the localization responses for the cancelled trials with the go and noncancelled trials (Fig. 2C). 

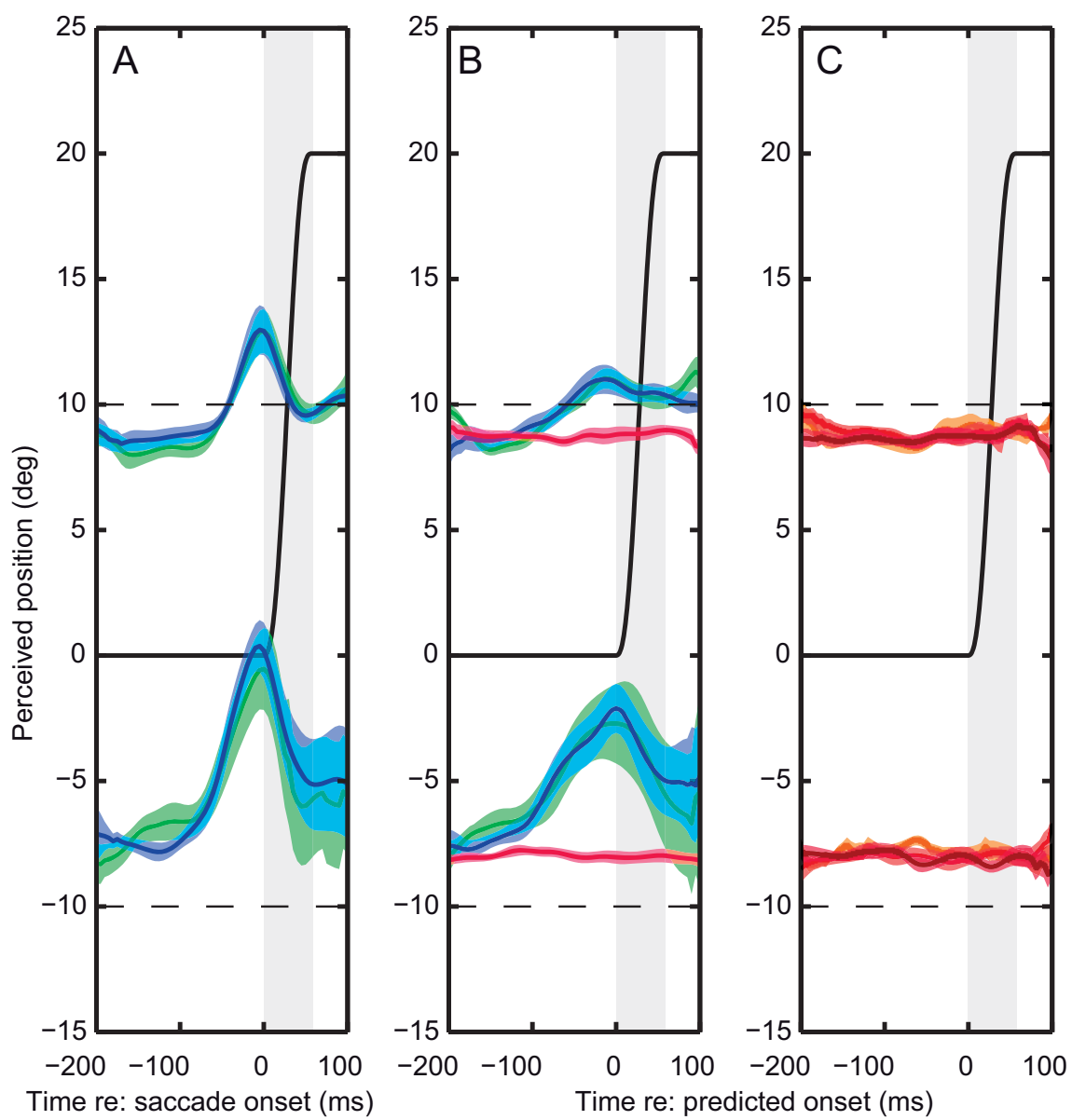

Time re: saccade onset (ms)

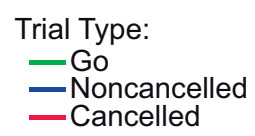

Cancelled Trials:

- Short SSD

- Medium SSD

Figure 3. Mean localization curves across all subjects. $A$, Localization curves of go and noncancelled trials, aligned to saccade onset. $\boldsymbol{B}$, Localization curves of go, noncancelled, and cancelled trials relative to predicted saccade onset. $\boldsymbol{C}$, Localization curves of cancelled trials do not differ for short, medium, and long SSDs $(p>0.05)$. Shaded areas, SEM.

For the go and noncancelled trials (green and blue, respectively) the mislocalization pattern shows a peak at saccade onset, resembling the pattern in Figure $2 A$ (the curves are not identical, given the impossibility of perfectly predicting saccade onset). In contrast, the successfully cancelled stop trials (red data points) indicate variable errors but no pattern, regardless of the location of the flash relative to the target. Thus, in contrast to the other trial types, flash localizations on cancelled stop trials do not show "perisaccadic" errors.

It is important to point out, however, that while the mean curves show no significant modulation, the localization data from cancelled trials may still contain some structure that should also be analyzed carefully to further validate this conclusion. We subdivided the cancelled stop trials into three categories based on the SSD. The rationale for this is that, on average, preparation will be more advanced for later SSDs before being inhibited. This contention is supported by neurophysiological evidence showing that some saccade-related neurons in FEF and SC ramp up to a higher level of activity before cancellation on stop trials with long versus short SSDs (Hanes et al., 1998; Paré and Hanes, 2003; Brown et al., 2008). The subdivision was done session by session (Fig. 2D). The subdivision groups contained approximately an equal amount of trials. If localization errors arose due to saccade preparation, we would predict larger mislocalization on cancelled trials with long SSDs. However, Figure $2 E$ shows overlapping mislocalization patterns for all three SSD categories that did not change over time.

Figure 3 summarizes the localization results of all eight subjects. Across subjects, the go trials (green) and noncancelled stop trials (blue) show highly overlapping curves (Fig. $3 A$ ). In Figure $3 B$, we show the same data relative to predicted saccade onset, together with the data of the cancelled trials, which resemble the observation of the cancelled trials for the single subject (Fig. 2C). Indeed, using a repeated-measures ANOVA, we found that localization errors are significantly influenced by flash location (inbound, outbound), trial type (go, noncancelled, cancelled), and time $[-150$ ms, 0 ms (predicted saccade onset; $F_{(1,7)}=$ 974.4, $p<0.0001 ; F_{(2,6)}=21.8, p<0.005$; and $F_{(1,7)}=41.3, p<0.0001$, respectively]. Importantly, the interaction between trial type and time was significant $\left(F_{(2,6)}=17.5, p<0.005\right)$, which means that the mislocalization effect is not identical for all three trial types. Post hoc testing revealed that localization changes significantly as a function of time for the go $\left(t_{(7)}=6.5, p<0.0001\right)$ and noncancelled trials $\left(t_{(7)}=6.2, p<0.0001\right)$, but not for the cancelled trials $\left(t_{(7)}=1.2, p>\right.$ $0.05 ; 95 \% \mathrm{CI},-0.10$ to $\left.0.31^{\circ}\right)$, which is consistent with the notion that the mislocalization effect occurs only when the saccade is executed. Because the CI is very small, the potential for a type II error (false negative) is low, which means that the present test would not be sensitive enough only if mislocalization is expected to be smaller than $0.31^{\circ}$. Furthermore, subdividing the cancelled trials into three SSD categories (Fig. $3 C$ ) does not reveal perisaccadic localization errors, even for long-SSD trials $\left(95 \% \mathrm{CI},-0.55\right.$ to $\left.0.34^{\circ}\right)$, where preparation is presumably most advanced. A repeated-measures ANOVA confirmed that although localizations of the inbound and outbound flashes were not identical $\left(F_{(1,7)}=1561.1, p<0.0001\right)$, neither the variable time $(-150 \mathrm{~ms}, 0 \mathrm{~ms})$ nor the variable SSD (short, medium, long) showed a significant effect.

Finally, as Figures 2 shows, localization responses also become more variable when the localization target was presented near the time of a saccade. Can saccade preparation alone at least increase the variability of localization of the flash? To test this, we repeated the same analyses, examining the variance of localization relative to saccade onset (variance was calculated using a sliding $40 \mathrm{~ms}$ bin and pooled over flash location). Figure 4 shows the variability in localization judgments across subjects. A repeated-measures ANOVA revealed no significant difference between the go and noncancelled trials $\left(F_{(1,7)}=0.1, p>0.05\right)$. Furthermore, variability changes significantly as a function of time for the go $\left(F_{(1,7)}=9.1, p<0.05\right)$ and noncancelled stop trials $\left(F_{(1,7)}=12.2\right.$, 
$p<0.05)$, but not for the cancelled trials $\left(F_{(1,7)}<0.001\right.$, n.s.; $95 \%$ CI, -0.79 to 0.78 $\left.\operatorname{deg}^{2}\right)$. Again, even after subdividing the stop trials (Fig. $4 C$ ), variability does not change over time $\left(F_{(1,7)}=0.4\right.$, n.s. $)$, and no significant differences exist between the three SSD categories $\left(F_{(2,6)}=1.2\right.$, $p>0.05)$.

\section{Discussion}

Briefly flashed objects are mislocalized around saccade onset. It has been suggested that planning an eye movement, not the saccade per se, could at least initiate this visual distortion. To test this hypothesis, we investigated the presence of visuospatial errors using a countermanding paradigm in which a planned saccade is suddenly aborted before its execution. Consistent with the literature, we found strong perisaccadic mislocalization when a saccade was executed, in both go trials as well as noncancelled stop trials. In contrast, no mislocalization pattern was found when a planned saccade was cancelled close to the point of no return. Similarly, the variability of localization errors was significantly smaller on cancelled compared to noncancelled saccades and did not change over time. Our results suggest that the actual execution of the saccade is a prerequisite for mislocalization of briefly flashed objects, rejecting the hypothesis that the preparation of saccade alone evokes such errors.

\section{Did subjects prepare a saccade in the cancelled trials?}

One potential criticism is that saccade preparation may not be far enough advanced on cancelled trials to test our hypothesis. However, a number of arguments suggest that the present paradigm pushed the saccadic system close to the point of no return. First, a saccadic sequence enhances saccade planning. Indeed, the reduced reaction time variability on sequences of saccades compared to regular saccades can be explained by a faster and less variable process of saccade preparation (Joiner et al., 2007). Second, the countermanding behavior we observed conformed to previous studies. Countermanding behavior is typically explained by a race model with stochastically independent accumulating GO and STOP processes, with saccade execution or cancellation being dictated by which process wins. In such a model, the GO process (essentially saccade preparation) may be quite advanced on successfully cancelled trials, depending on the SSD and the progression of the STOP process. Although such an independent race model is not entirely consistent with the neurophysiology of the oculomotor system, where gazeholding and gaze-shifting mechanisms can interact, computational "interactive" race models show that cancelled trials can still feature highly advanced saccade preparation before potent cancellation (Boucher et al., 2007). Third, a common response when the head is unrestrained is the "head-only movement," where preparation on successfully cancelled gaze shifts is still advanced enough to initiate an orienting head movement to the target (Corneil and Elsley, 2005; Goonetilleke et al., 2010). Head-only movements are most common at the intermediate SSDs that are preferentially sampled by the one-up, onedown method of determining SSD used here (Corneil et al., 2013), as this method best balances the GO and STOP processes against each other. Fourth, neurophysiological studies of saccade countermanding show that some saccade-related neurons in the SC and FEF display substantial buildup of activity on cancelled saccades before being abruptly curtailed before saccade execution (Hanes et al., 1998; Paré and Hanes, 2003). In fact, buildup activity in these areas is essentially identical for both cancelled and noncancelled saccades up until $\sim 40$ ms before saccade execution, at which point the activity for cancelled saccades is abruptly curtailed. Thus, even though we cannot know the degree of preparation on a trial-to-trial basis, we can infer that preparation was very advanced, and sometimes just a few tens of milliseconds away from a saccade, especially when the SSD was long.

\section{Why was there no mislocalization effect with cancelled saccades?}

The current findings show that even with substantial preparation, no trace of perisaccadic mislocalization arises if the saccade is ultimately cancelled. Whereas the mislocalization effect could easily exceed $5^{\circ}$ with noncancelled saccades, the confidence intervals for cancelled saccades is less than one-third of a degree anywhere, indicating that any substantial mislocalization effect in those trials can be ruled out. We consider this a surprising finding given that saccade preparation increases visuospatial sensitivity at the saccade target area (Zhao et al., 2012). This modulation is presumably caused by the buildup of activity on saccade-related neurons in the SC and FEF, which may project via reciprocal connections to posterior extrastriate areas like LIP, V4, V3, and V2 (Huerta et al., 1987; Schall, 1995; Stanton et al., 1995). Microstimulation in FEF with a current insufficient to evoke saccades nevertheless increases sensitivity in visual cortex at the retinotopic coordinates where the eyes would have been otherwise guided (Moore et al., 2003). The time course of these presaccadic modulations resembles the dynamics of mislocalization around a saccade (Hamker et al., 2008; Burr and Morrone, 2010). Therefore, it has been argued that although this presaccadic sensitivity at the saccade target may be critical for efficient visual processing (and/or visual stability), it distorts the memorized distance between the saccade target and a flashed object, producing perisaccadic mislocalization (Lappe et al., 2000; Hamker et al., 2008, 2011). The distortion of distance arises because the weak position 
signal of the flash is averaged with the very strong position signal of the saccade target, which subsequently "pulls" the flash toward it. This explanation is also consistent with the idea that receptive fields stretch or shift perisaccadically, which is in turn responsible for perisaccadic mislocalization (Ross et al., 2001; Tolias et al., 2001; Kusunoki and Goldberg, 2003; Burr and Morrone, 2010; Cicchini et al., 2013). So, why was there no mislocalization effect with cancelled saccades?

Our results show that mislocalization only arises when the saccade is actually executed. If we assume that perisaccadic mislocalization reflects the transsaccadic remapping of object information (which might be carried out by shifting receptive fields), the current findings suggest that the remapping starts only when the saccade plan cannot be aborted anymore. This is consistent with the suggestion by Sommer and Wurtz (2008), who argued receptive fields should shift only if the generation of the saccade is inevitable. Viewed from the other end, if remapping is performed every time we plan a saccade, instability would arise when the plan is aborted before execution, which seems like a suboptimal mechanism.

Taking this one step further, it could be suggested from our results that those saccade-related neurons in SC and FEF that do not distinguish between cancelled and noncancelled trials during their buildup play no major role in the neural mechanisms for visual stability. Interestingly, Ray et al. (2009) showed that visuomovement neurons in the FEF, which are another functional class of saccade-related neuron, peak in activity only when a saccade is truly inevitable. The authors speculated that this late enhancement begins at a time coinciding with the transition from controlled to ballistic saccade programming, perhaps only then contributing to the update of visual representations associated with the saccade. This functional distinction between saccaderelated neurons could explain our results, and an anatomical basis for segregation may exist (Pouget et al., 2009). Linking to the ideas discussed in the previous paragraph, the encoded distance between the saccade target and flashed object may become distorted only when these visuomovement neurons burst.

\section{Alternative explanations of the present results}

Although the present findings provide a novel view on the saccadic remapping mechanisms for visual stability, there are other explanations that should be considered. Previously, Maij et al. (2011b, F. Maij, E. Brenner, J. B. J. Smeets, and R. J. van Beers, unpublished observations) provided an optimal integration model, explaining perisaccadic mislocalization as the result of uncertainty in the time of the flash combined with a foveal bias. Their rationale is that perisaccadic mislocalization occurs because the observer is uncertain about the time of the flash relative to the saccade and has a prior expectation that any perceived flash must have been close to the fovea. When no saccade is executed there is no ambiguity of where the eyes were at the time of the flash; hence no perisaccadic mislocalization would occur. This would also be consistent with the idea that presaccadic buildup activity in FEF and SC does not play a role in perisaccadic mislocalization.

There are also suggestions that perisaccade mislocalization effects are unrelated to making saccades at all. Ostendorf et al. (2006) compared flash localization in a condition where a $10^{\circ}$ saccade was executed with that in a condition in which the subject kept fixation but the stimulus display was moved $10^{\circ}$ in a fast saccade-like fashion. Mislocalization in the latter condition had a magnitude and time course comparable to those in the saccade condition. Another previous study, conducted by Zimmermann et al. (2013), reported a strong compression of space around a visual anchor. While subjects kept fixation, the anchor was presented, followed by a brief whole-field mask. An object flashed around the time of the mask was mislocalized in the direction of the anchor.

Both these studies suggest that neither the preparation nor the execution of the saccade is a prerequisite for perisaccadic-like distortions of space. With an actual saccade, a masking effect is provided by the retinal motion blur, which is not present with saccade planning alone. Based on these results, it can be suggested that when a transient object is presented, the distance from this object toward the currently attended location is distorted in memory. This distorted representation is used only after the occurrence of a visual discontinuity, such as a saccade, mask, or stimulus motion. When no visual discontinuity occurs, localization can be carried out on a purely retinal basis without "scene reconstruction," yielding veridical localization. Future research should be conducted to test this proposal.

Finally, the conceptual approach of the present study could be extended to the study of other perisaccadic phenomena, including saccadic suppression, sluggish internal representations of eye position, or remapping. The abrupt cancellation of saccades just before saccade execution could advance the understanding of whether a given behavioral or neurophysiological phenomenon is driven by saccade preparation or not, independent of perisaccadic or postsaccadic processes. Our results suggest that perisaccadic mislocalization is contingent on saccade execution or transsaccadic memory and hence helps to constrain the involved neural substrates.

\section{References}

Awater H, Lappe M (2006) Mislocalization of perceived saccade target position induced by peri-saccadic visual stimulation. J Neurosci 22:12-20. Medline

Boucher L, Palmeri TJ, Logan GD, Schall JD (2007) Inhibitory control in mind and brain: an interactive race model of countermanding saccades. Psychol Rev 114:376-397. CrossRef Medline

Brown JW, Hanes DP, Schall JD, Stuphorn V (2008) Relation of frontal eye field activity to saccade initiation during a countermanding task. Exp Brain Res 190:135-151. CrossRef Medline

Burr DC, Morrone MC (2010) Vision: keeping the world still when the eyes move. Curr Biol 20:442-444. CrossRef

Cicchini GM, Binda P, Burr DC, Morrone MC (2013) Transient spatiotopic integration across saccadic eye movements mediates visual stability. J Neurophysiol 109:1117-1125. CrossRef Medline

Corneil BD, Elsley JK (2005) Countermanding eye-head gaze shifts in humans: marching orders are delivered to the head first. J Neurophysiol 94:883-895. CrossRef Medline

Corneil BD, Cheng JC, Goonetilleke SC (2013) Dynamic and opposing adjustment of movement cancellation and generation in an oculomotor countermanding task. J Neurosci 33:9975-9984. CrossRef Medline

Duhamel JR, Colby CL, Goldberg ME (1992) The updating of the representation of visual space in parietal cortex by intended eye movements. Science 255:90-92. CrossRef Medline

Goonetilleke SC, Doherty TJ, Corneil BD (2010) A within trial measure of the stop signal reaction time in a head-unrestrained oculomotor countermanding task. J Neurophysiol 104:3677-3690. CrossRef Medline

Hamker FH (2005) The reentry hypothesis: the putative interaction of the frontal eye field, ventrolateral prefrontal cortex, and areas V4, IT for attention and eye movement. Cereb Cortex 15:431-447. CrossRef Medline

Hamker FH, Zirnsak M, Calow D, Lappe M (2008) The peri-saccadic perception of objects and space. PLoS Comput Biol 4:e31. CrossRef Medline

Hamker FH, Zirnsak M, Ziesche A, Lappe M (2011) Computational models of spatial updating in peri-saccadic perception. Philos Trans R Soc Lond B Biol Sci 366:554-571. CrossRef Medline

Hanes DP, Patterson WF 2nd, Schall JD (1998) Role of frontal eye fields in 
countermanding saccades: visual, movement, and fixation activity. J Neurophysiol 79:817-834. Medline

Huerta MF, Krubitzer LA, Kaas JH (1987) Frontal eye field as defined by intracortical microstimulation in squirrel monkeys, owl monkeys, and macaque monkeys. II. Cortical connections. J Comp Neurol 265:332-361. CrossRef Medline

Joiner WM, Lee JE, Shelhamer M (2007) Behavioral analysis of predictive saccade tracking as studied by countermanding. Exp Brain Res 181:307320. CrossRef Medline

Kusunoki M, Goldberg ME (2003) The time course of peri-saccadic receptive field shifts in the lateral intraparietal area of the monkey. J Neurophysiol 89:1519-1527. Medline

Lappe M, Awater H, Krekelberg B (2000) Postsaccadic visual references generate presaccadic compression of space. Nature 403:892-895. CrossRef Medline

Logan GD (1994) On the ability to inhibit thought and action: a users' guide to the stop signal paradigm. In: Inhibitory processes in attention, memory, and language (Dagenbach D, Carr TH, eds), pp 189-239. San Diego: Academic.

Logan GD, Cowan WB (1984) On the ability to inhibit thought and action: a theory of an act of control. Psychol Rev 91:295-327. CrossRef

Maij F, Brenner E, Smeets JBJ (2011a) Peri-saccadic mislocalization is not influenced by the predictability of the saccade target location. Vision Res 51:154-159. CrossRef Medline

Maij F, Brenner E, Smeets JBJ (2011b) Temporal uncertainty separates flashes from their background during saccades. J Neurosci 31:3708-3711. CrossRef Medline

Michels L, Lappe M (2004) Contrast dependency of saccadic compression and suppression. Vision Res 44:2327-2336. CrossRef Medline

Moore T, Tolias AS, Schiller PH (1998) Visual representations during saccadic eye movements. Neurobiology 95:8981-8984. Medline

Moore T, Armstrong KM, Fallah M (2003) Visuomotor origins of covert spatial attention. Neuron 40:671-683. CrossRef Medline

Morris AP, Kubischik M, Hoffmann KP, Krekelberg B, Bremmer F (2012) Dynamics of eye-position signals in the dorsal visual system. Curr Biol 22:173-179. CrossRef Medline

Nakamura K, Colby CL (2002) Updating of the visual representation in monkey striate and extrastriate cortex during saccades. Proc Natl Acad Sci U S A 99:4026:4026-4031. Medline

Ostendorf F, Fischer C, Gaymard B, Ploner CJ (2006) Peri-saccadic mislocalization without saccadic eye movements. Neuroscience 137:737-745. CrossRef Medline

Paré M, Hanes DP (2003) Controlled movement processing: superior colliculus activity associated with countermanded saccades. J Neurosci 23: 6480-6489. Medline

Pouget P, Stepniewska I, Crowder EA, Leslie MW, Emeric EE, Nelson MJ,
Schall JD (2009) Visual and motor connectivity and the distribution of calcium-binding proteins in macaque frontal eye field: implications for saccade target selection. Front Neuroanat 3:1-14. CrossRef

Ray S, Pouget P, Schall JD (2009) Functional distinction between visuomovement and movement neurons in macaque frontal eye field during saccade countermanding. J Neurophysiol 102:3091-3100. CrossRef Medline

Richard A, Churan J, Guitton DE, Pack CC (2009) The geometry of perisaccadic visual perception. J Neurosci 29:10160-10170. CrossRef Medline

Ross J, Morrone MC, Burr DC (1997) Compression of visual space before saccades. Nature 386:598-601. CrossRef Medline

Ross J, Morrone MC, Goldberg ME, Burr DC (2001) Changes in visual perception at the time of saccades. Trends Neurosci 24:113-121. CrossRef Medline

Schall JD (1995) Neural basis of saccade target selection. Rev Neurosci 6:6385. Medline

Schall JD, Godlove DC (2012) Current advances and pressing problems in studies of stopping. Curr Opin Neurobiol 22:1012-1021. CrossRef Medline

Sommer MA, Wurtz RH (2008) Brain circuits for the internal monitoring of movements. Annu Rev Neurosci 31:317-338. CrossRef Medline

Stanton GB, Bruce CJ, Goldberg ME (1995) Topography of projections to posterior cortical areas from the macaque frontal eye fields. J Comp Neurol 353:291-305. CrossRef Medline

Tolias AS, Moore T, Smirnakis SM, Tehovnik EJ, Siapas AG, Schiller PH (2001) Eye movements modulate visual receptive fields of V4 neurons. Neuron 29:757-767. CrossRef Medline

Umeno MM, Goldberg ME (1997) Spatial processing in the monkey frontal eye field. I. Predictive visual responses. J Neurophysiol 78:1373-1383. Medline

von Helmholtz H (1925) Helmholtz's treatise on physiological optics, 3rd ed (Southall JP, transl.). New York: Optical Society of America.

Walker MF, Fitzgibbon EJ, Goldberg ME (1995) Neurons in the monkey superior colliculus predict the visual results of impending saccadic eyemovements. J Neurophysiol 73:1988-2003. Medline

Wurtz RH, Joiner WM, Berman RA (2011) Neuronal mechanisms for visual stability. progress and problems. Philos Trans R Soc Lond B Biol Sci 366:492-503. CrossRef Medline

Zhao M, Gersch TM, Schnitzer BS, Dosher BA, Kowler E (2012) Eye movements and attention: the role of pre-saccadic shifts of attention in perception, memory and the control of saccades. Vision Res 74:40-60. CrossRef Medline

Zimmermann E, Fink G, Cavanagh P (2013) Perifoveal spatial compression. J Vis 13(5):21 1-9. CrossRef 